\title{
Factores asociados con el cumplimiento de las precauciones de contacto en un hospital de alto nivel de complejidad*
}

\author{
Factors related to contact-warnings compliance in a high complexity hospital
}

Fatores associados à adesão às precauções de contato em um hospital de alto nível de complexidade

\author{
Laura Arango Castrillón ${ }^{\text {a }}$ \\ Hospital Universitario San Vicente Fundación, Colombia \\ laura.arango@sanvicentefundacion.com \\ ORCID: https://orcid.org/0000-0003-3602-965X
}

DOI: https://doi.org/10.11144/Javeriana.ie23.facp

Recibido: 19 Abril 2021

Aceptado: 17 Noviembre 2021

Publicado: 30 Diciembre 2021

\section{Resumen:}

Introducción: La prevención de infecciones intrahospitalarias es uno de los aspectos más inquietantes en enfermería en los últimos años. Para esto, se han establecido diversas estrategias, entre ellas, los aislamientos hospitalarios. Sin embargo, se encuentra gran diAcultad para el cumplimiento de las medidas de aislamiento. Objetivo: Determinar los factores sociodemográAcos y de la atención en salud relacionados con el cumplimiento de las precauciones de contacto por parte de los visitantes en un hospital de alta complejidad de la ciudad de Medellín. Método: Se realizó un estudio descriptivo de corte trasversal a un total de 126 visitantes de pacientes aislados por contacto. Se identiAcaron factores asociados al cumplimiento del aislamiento mediante una encuesta, se analizó la información en el programa SPSS mediante una regresión logística y se asumió signiacancia estadística si valor P menor a 0,05. Resultados: La adherencia total al aislamiento fue de un 40,5\% y está asociada principalmente con dos factores: disponibilidad de los elementos de protección y estar de acuerdo con el uso de estos. Conclusiones: Los factores mejor asociados con el cumplimiento del aislamiento hacen necesario que las instituciones garanticen suAcientes insumos para los visitantes y que tanto el personal de salud como los visitantes reciban una mayor sensibilización en este tema.

Palabras clave: aislamiento de pacientes, adherencia al tratamiento, precauciones universales.

\section{Abstract:}

Introduction: Preventing the intra-hospital infections is one of the major concerns in the last year. To that end, different strategies have been set such as hospital isolation. However, there is a grate default in order to comply with the isolation measures Objective: To determine the sociodemographic and healthcare factors related to how to comply with the contact warnings by the visitors coming into a high complexity hospital in Medellín. Methods: It is a cross-sectional descriptive study including in total 126 visitors who were isolated aÆer a contact. Factors associated with the isolation compliance were identiaed based on a survey. Information was analyzed with SPSS soÆware by doing a logistic regression and statistical signiAcance was assumed as $\mathrm{P}<0.05$. Results: Total adherence to isolation was $40.5 \%$, which is mainly associated with two factors: availability of protective garments and to agree with their use. Conclusions: ée factors deemed as best associated with the isolation compliance required that the institutions provided enough protective garments to the visitors and that both the visitors and the hospital staff can receive a better awareness-rising communication on this topic.

Keywords: patient isolation, treatment adherence, universal warnings.

\section{Resumo:}

Introdução: A prevenção de infeções intrahospitalares é um dos aspectos mais preocupantes nos últimos anos. Para isso, tem se estabelecido várias estratégias, entre elas, o isolamento hospitalar. No entanto, há grande diaculdade para a adesão as medidas de isolamento. Objetivo: Determinar os fatores sociodemográAcos e da atenção em saúde relacionados com o cumprimento das precauções de contato por parte dos visitantes em um hospital de alta complexidade na cidade de Medellín. Método: Foi realizado um estudo descritivo transversal com um total de 126 visitantes de pacientes isolados por contato. IdentiAcaram-se fatores associado ao cumprimento do isolamento mediante inquérito, analisou-se a informação no programa SPSS mediante regressão logística e foi assumida signiAcância estatística se o valor P fosse menor a 0,05. Resultados: A adesão total ao isolamento foi de 40,5\% e está associada principalmente com dois fatores: disponibilidade dos elementos de proteção e concordância com seu uso. Conclusões: Os

Notas de autor

${ }^{\text {a }}$ Autora de correspondencia: laura.arango@sanvicentefundacion.com 
fatores mais bem associados ao cumprimento do isolamento tornam necessário que as instituições garantam suprimentos suAcientes para os visitantes e que tanto o pessoal de saúde quanto os visitantes recebam maior conscientização sobre essa questão.

Palavras-chave: isolamento de pacientes, adesão ao tratamento, precauções universais.

\section{Introducción}

En la prevención de las infecciones asociadas con la atención en salud (IAAS, juega un papel muy importante tanto el personal de la salud como el mismo paciente, sus visitantes y familiares. Estas infecciones son conocidas, según la guía de precauciones de aislamiento del CDC (centro para el control y prevención de enfermedades de los Estados Unidos, como aquellas no solo adquiridas en el hospital (Infección nosocomial, sino las asociadas con la prestación de la atención médica en cualquier entorno, ya sea hospital, centro de salud, atención domiciliaria entre otros (1.

Estas infecciones han elevado los costos de la atención en salud y la mortalidad de las personas. En un artículo publicado en 2015 en Bogotá, Colombia, donde evaluaron 196 pacientes de un hospital universitario de esta ciudad, encontraron que la mortalidad atribuible a las IAAS fue del 26,4\%; el exceso de costos atribuible a las IAAS, en general, fue de US\$ 5,122. El exceso de días de estancia hospitalaria atribuible a estas fue de 16 días. Además de esto, dichas infecciones aumentaron el consumo de medicamentos y ayudas diagnósticas. (2)

El control de las IAAS se ha vuelto, entonces, cada vez más importante. Según la OMS, están apareciendo nuevos mecanismos de resistencia a los antimicrobianos que ponen en peligro la capacidad de las personas para tratar enfermedades infecciosas comunes, lo que aumenta la discapacidad, las muertes y la prolongación de la enfermedad $(3$.

En un hospital de Barcelona, la mortalidad de los pacientes con bacterias patógenas multirresistentes, clasiacadas como colonización, fue del $63,4 \%$, mientras que la de los clasiacados como infección fue del 64,2 \%; a pesar de esto, al evaluar la eAcacia de las precauciones de aislamiento, dicho estudio mostró que los servicios incluidos no presentaron ningún brote epidémico por estas bacterias, lo que se atribuye a que el cumplimiento de las precauciones de contacto limite la diseminación de estos microorganismos (4).

Respecto a lo anterior, la OPS aArma que los programas de prevención y control de infecciones han demostrado su beneacio (5). Dentro de estos programas están las precauciones de aislamiento deanidas como un conjunto de acciones que permiten la separación de pacientes durante el periodo de transmisibilidad de la enfermedad, lo que permite cortar la cadena de infección (6). Uno de los trabajos que motivan a esto es el de Cohen CC y Col. en Estados Unidos, que aconseja continuar invirtiendo en estudios para mejorar los componentes de las precauciones de contacto, en busca de la prevención de infecciones (7).

A pesar de que muchos de los visitantes de los pacientes conocen las precauciones de aislamiento y la función en la prevención de la transmisión de microorganismos asegurar que las cumplan es un desafío en la atención médica (8).

A nivel Internacional, se ha encontrado incumplimiento del aislamiento de contacto por los familiares hasta del $23,7 \%$ en el uso de bata y guantes, además los recursos para el aislamiento están casi universalmente disponibles en los hospitales, pero se encuentran infrautilizados (9).

En Colombia, una investigación realizada en la IPS universitaria de Medellín, concluyó que los familiares y visitantes no tienen una total adherencia a las técnicas de aislamiento. Entre las técnicas que más se incumplen están el uso correcto de la mascarilla de alto Áujo (incumplimiento del 60,3\%) y la restricción de visitas (10).

De manera similar, en un hospital de alta complejidad de Medellín — lugar donde se realizó el estudio-se hace una evaluación transversal a las medidas de aislamiento hospitalario. En el indicador del año 2019 se encontró incumplimiento a los aislamientos por los visitantes del 31,82\%, una proporción de salas con los implementos necesarios del $96,43 \%$ y una proporción de pacientes que conocen el tipo de aislamiento de 
95,08\%. El aislamiento de contacto es el que mayormente se presenta en este hospital y en el que se enfocó la investigación $(11,12$

La importancia del control de estas infecciones radica en que acarrean grandes costos en el tratamiento de los pacientes y consecuencias graves en su recuperación. Sin embargo, el enfoque preventivo se ha centrado en las acciones del personal de la salud, dejando de lado los otros integrantes de las cadenas de transmisión. Así es comentado por la guía de expertos de la SHAE (Sociedad de epidemiología del cuidado a la salud de américa, quienes promueven que se realicen nuevas investigaciones con las que se puedan determinar datos certeros acerca de la adherencia de los visitantes a los aislamientos hospitalarios, sus factores relacionados e implicaciones en el no cumplimiento (8.

Por lo anterior, el objetivo de este estudio es determinar los factores sociodemográacos y de la atención en salud asociados con el cumplimiento de las precauciones de aislamiento por contacto por parte de los visitantes en un hospital de alta complejidad de la ciudad de Medellín.

\section{Método}

Se realizó un estudio descriptivo de corte transversal. La población estuvo constituida por los visitantes de los pacientes aislados de todo tipo contacto desde enero a julio de 2020. Se realizó un muestreo no probabilístico, a conveniencia. No se deAnió previamente un tamaño de muestra, sino que se incluyeron todos los visitantes presentes durante el periodo de la recolección de los datos. En total hubo 126 participantes. Se incluyeron los visitantes mayores de 18 años que voluntariamente aceptaron participar en el estudio y se excluyeron los visitantes de pacientes con aislamiento en urgencias o servicios de apoyo y pacientes con infección respiratoria por SARS-COV-2.

En cuanto al instrumento, la recolección de la información se hizo mediante una encuesta realizada de forma presencial por la investigadora y los auxiliares de investigación previa capacitación de ellos. Esta contaba con 15 preguntas cerradas en su mayoría dicotómicas (sí, no. Dentro de estas, la variable desenlace, cumplimiento del aislamiento, tenía cuatro preguntas que reÁejaban este aspecto (la falla de alguna de estas indicaba no cumplimiento del aislamiento y diez preguntas sobre variables de exposición que pueden favorecer o no el aislamiento. Todas las preguntas fueron elaboradas con base en el instrumento utilizado por el área de epidemiología del hospital para la evaluación de la adherencia a los aislamientos hospitalarios. Recomendaciones del CDC ( 1 y la SHAE (8, estudios donde evalúan la adherencia del personal, como el caso de multirresistentes en España ( 9 y la experiencia personal, no se encontró ninguna encuesta validada para los visitantes. La encuesta fue modiAcada, en algunas de sus variables, por una prueba piloto que se realizó previamente a cinco visitantes.

Se indagó en el servicio de epidemiología sobre la cantidad aproximada de pacientes que se encontraban en aislamiento de contacto. Se visitaron los servicios durante varios días a la semana en el mes de enero a julio del año 2020, para realizarle la encuesta al acompañante que estuvo presente en el momento de la visita, a excepción de los pacientes que, por su condición, nunca tenían acompañante o presentaban algún criterio de exclusión.

La información recolectada se procesó en el programa $S P S S^{\circledR}$ versión 22; luego, se revisó en busca de valores erróneos para ajustarlos. Las variables cualitativas se describen mediante frecuencias absolutas y relativas. La variable cuantitativa edad no distribuye normal según la prueba de normalidad de Shapiro Wilk, por ello se describe su mediana y rango intercuartílico. Dada la naturaleza y el nivel de medición de la variable desenlace, que fue cualitativa dicotómica (Cumplimiento Sí o No, se realizó para el análisis de los factores asociados la prueba CHI cuadrado y estimación de OR crudos y OR ajustados mediante regresión logística, teniendo cuenta valor $\mathrm{P}<0,05$ se dejaron solo 2 variables que demostraron estar asociadas con el desenlace: disponibilidad de los elementos de protección y estar de acuerdo con el aislamiento. 
El estudio tuvo la aprobación del comité de ética e investigación y fue clasiacado como una investigación sin riesgo según la resolución 8430/1993. La encuesta que se realizó fue de manera anónima y previa aprobación del consentimiento informado del participante. Este trabajo se realizó en busca de la prevención en la transmisión de infecciones cruzadas, por lo que se conservaron así los principios éticos de la declaración de Helsinki para la investigación en seres humanos.

\section{Resultados}

La distribución porcentual de la población estudiada, según sus características sociodemográAcas, mostró que el 78,6\% de la población son mujeres. La mediana de la edad de las personas estudiadas fue de 42 años, con un rango intercuartílico de 22 años. La edad mínima fue de 18 años y la máxima de 72 años. Dentro del estrato socioeconómico, el 32,5\% de los participantes era estrato 3, el 81\% tenía estudios hasta secundaria o menos y, según los rangos de edad, los adultos de 27 a 72 años corresponden al 86,5\% de dicha población.

Se analizaron seis factores relacionados con la atención en salud que se asocian con el cumplimiento de los aislamientos. Entre ellos, se encontró que el 94\% de los visitantes maniAesta conocer el aislamiento y elementos que se utilizan en él, el $81 \%$ encontró disponibles todos los elementos de protección, el 93,7\% conoce la restricción de visitas, el 91,3\% dijo estar de acuerdo con el aislamiento, al 71,3\% le fue explicado el aislamiento por el personal de enfermería y el 52,4\% cuando ingresaron al servicio (ver tabla No. 1).

TABLA NO. 1

Factores relacionados con la atención en salud

\begin{tabular}{|c|c|c|}
\hline \multicolumn{2}{|c|}{ Factores relacionados con la atención en salud de los visitantes } & \multirow{2}{*}{\begin{tabular}{|l|l|}
$\mathbf{N}=\mathbf{l 2 6}$ \\
$\mathbf{F a}(\%)$
\end{tabular}} \\
\hline \multirow{2}{*}{$\begin{array}{l}\text { Conocer el aislamiento y elementos que } \\
\text { se utilizan }\end{array}$} & Sí & \\
\hline & No & $7(5,6)$ \\
\hline \multirow{2}{*}{$\begin{array}{l}\text { Disponibilidad de todos los elementos de } \\
\text { protección }\end{array}$} & Sí & $102(81)$ \\
\hline & No & $24(19)$ \\
\hline \multirow{2}{*}{$\begin{array}{l}\text { Estar de acuerdo con el uso de los } \\
\text { elementos de protección }\end{array}$} & Sí & $115(91,3)$ \\
\hline & No & $11(8,7)$ \\
\hline \multirow{2}{*}{$\begin{array}{l}\text { Conocer la restricción de visitas al } \\
\text { paciente }\end{array}$} & Sí & $118(93,7)$ \\
\hline & No & $8(6,3)$ \\
\hline \multirow[t]{5}{*}{ Persona que le explicó el Aislamiento } & Personal de enfermería & $90(71,4)$ \\
\hline & Personal médico & $15(11,9)$ \\
\hline & \begin{tabular}{|l|} 
Otro familiar \\
\end{tabular} & $16(12,7)$ \\
\hline & El paciente & $4(3,2)$ \\
\hline & No le explicaron & $1(0,8)$ \\
\hline \multirow{4}{*}{$\begin{array}{l}\text { Momento en que se le explicó el } \\
\text { Aislamiento }\end{array}$} & $\mathrm{Al}$ ingresar al servicio & $66(52,4)$ \\
\hline & Durante la hospitalización & $41(32,5)$ \\
\hline & En otro momento & $18(14,3)$ \\
\hline & No se le explicó & $1(0,8)$ \\
\hline
\end{tabular}

Fuente: Elaboración propia

Dentro de la variable dependiente cumplimiento a las medidas de aislamiento, el 95,2\% conocían el lavado de manos como principal precaución; el 68\% tenía la bata y guantes al momento de la encuesta; el $93,7 \%$ permanecía con la bata y guantes dentro de la habitación y el $42,9 \%$ no se retiró los guantes dentro de la habitación. Se tiene un cumplimiento total del aislamiento con estas 4 variables del 40,5\%, esta variable compuesta es la variable dependiente (ver tabla 2).

TABLA NO. 2

Factores que determinan la adherencia a las medidas de aislamiento

\begin{tabular}{|l|l|l|}
\hline \multicolumn{2}{|l|}{ Factores que determinan el cumplimiento del aislamiento } & $\begin{array}{l}\text { N=126 } \\
\text { Fa (\%) }\end{array}$ \\
\hline Lavado de manos como principal precaución & Sí & $120(95,2)$ \\
\cline { 2 - 3 } & No & $6(4,8)$ \\
\hline \multirow{2}{*}{$\begin{array}{l}\text { Bata y guantes puestos al momento de la } \\
\text { encuesta }\end{array}$} & Sí & $68(54)$ \\
\cline { 2 - 3 } & No & $58(46)$ \\
\hline Permanece afuera de la habitación con la bata & Sí & $8(6,3)$ \\
\cline { 2 - 3 } o guantes & No & $118(93,7)$ \\
\hline \multirow{2}{*}{$\begin{array}{l}\text { Ausencia de la bata o guantes puestos dentro } \\
\text { de la habitación }\end{array}$} & Sí & $72(57,1)$ \\
\cline { 2 - 3 } & No & $54(42,9)$ \\
\hline Adherencia al total de medidas de aislamiento & Sí & $51(40,5)$ \\
\cline { 2 - 3 } & No & $75(59,5)$ \\
\hline
\end{tabular}

Fuente: Elaboración propia. 
En cuanto a las asociaciones entre variables sociodemográAcas y el cumplimiento del aislamiento, no se encontraron diferencias estadísticamente signiacativas. Sin embargo, el género con un OR: 1,49 (IC: 0,633-3,511) da entender que es un $49 \%$ más frecuente que las mujeres cumplan con el aislamiento y la escolaridad con OR: 0,502 (0,205-1,233) muestra que, independientemente del nivel educativo que se tenga, hay una probabilidad similar para el cumplimiento del aislamiento (ver tabla No. 3).

TABLA NO. 3

Asociación del cumplimiento del aislamiento con las variables sociodemográAcas.

\begin{tabular}{|c|c|c|c|c|c|c|c|c|}
\hline \multirow{3}{*}{\multicolumn{2}{|c|}{ Variables Sociodemográficas }} & \multicolumn{4}{|c|}{$\begin{array}{lrr}\begin{array}{l}\text { Cumplimiento } \\
\text { medidas }\end{array} & \begin{array}{r}\text { las } \\
\text { de }\end{array} \\
\text { aislamiento } & & \\
\end{array}$} & \multirow[t]{3}{*}{ OR } & \multirow[t]{3}{*}{$\begin{array}{l}\text { Valor } \\
\text { de P }\end{array}$} & \multirow[t]{3}{*}{$\begin{array}{l}\text { IC } \\
95 \%\end{array}$} \\
\hline & & \multicolumn{2}{|c|}{ Sí n=51 } & \multicolumn{2}{|c|}{ No $n=75$} & & & \\
\hline & & $\mathbf{N}$ & $\%$ & $\mathbf{N}$ & $\%$ & & & \\
\hline \multirow{2}{*}{$\begin{array}{l}\text { Estrato } \\
\text { Socioeconómic } \\
\text { o }\end{array}$} & Estrato 4 al 6 & 3 & 5,9 & 2 & 2,7 & \multirow[t]{2}{*}{0,438} & \multirow{2}{*}{$\begin{array}{l}0,323 \\
*\end{array}$} & \multirow{2}{*}{$\begin{array}{l}0,071- \\
2,721\end{array}$} \\
\hline & Estrato 0 al 3 & 48 & 94,1 & 73 & 97,3 & & & \\
\hline \multirow[t]{2}{*}{ Escolaridad } & Educación Superior & 13 & 25,5 & 11 & 14,7 & \multirow[t]{2}{*}{0,502} & \multirow[t]{2}{*}{0,129} & \multirow{2}{*}{\begin{tabular}{|l|}
$0,205-$ \\
1,233 \\
\end{tabular}} \\
\hline & Secundaria o menos & 38 & 74,5 & 64 & 85,3 & & & \\
\hline \multirow[t]{2}{*}{ Género } & Mujer & 38 & 74,5 & 61 & 81,3 & \multirow[t]{2}{*}{1,49} & \multirow[t]{2}{*}{0,360} & \multirow{2}{*}{$\begin{array}{l}0,633- \\
3,511\end{array}$} \\
\hline & Hombre & 13 & 25,5 & 14 & 18,7 & & & \\
\hline \multirow[t]{2}{*}{ Edad } & Mayor a 27 años & 44 & 86,3 & 65 & 86,7 & \multirow[t]{2}{*}{1,034} & \multirow[t]{2}{*}{0,950} & \multirow{2}{*}{$\begin{array}{l}0,366- \\
2,922\end{array}$} \\
\hline & De 0 a 26 años & 7 & 13,7 & 10 & 13,3 & & & \\
\hline
\end{tabular}

* Se eligió prueba exacta de Fisher por recuento menor que 5 en más de 1 casilla. Fuente: Elaboración propia.

Para las variables relacionadas con la atención en salud, solo se encontró estadísticamente la disponibilidad de los elementos de protección, lo que dio a entender que hay 3,1 probabilidades de que, al tener estos elementos de protección, se cumpla con el aislamiento. En cuanto a la variable: persona que explica el aislamiento, el cumplimiento de este requisito es un 34\% más frecuente cuando lo explica el personal de enfermería que cuando lo explica otro familiar, el mismo paciente o no le fue explicado el aislamiento. Para la variable: momento en que se explicó el aislamiento, es un 19\% más frecuente que se cumpla el aislamiento si la explicación fue al ingreso del servicio que en otro momento. (Ver tabla 4).

TABLA 4

Asociación con las variables relacionadas con la atención en salud

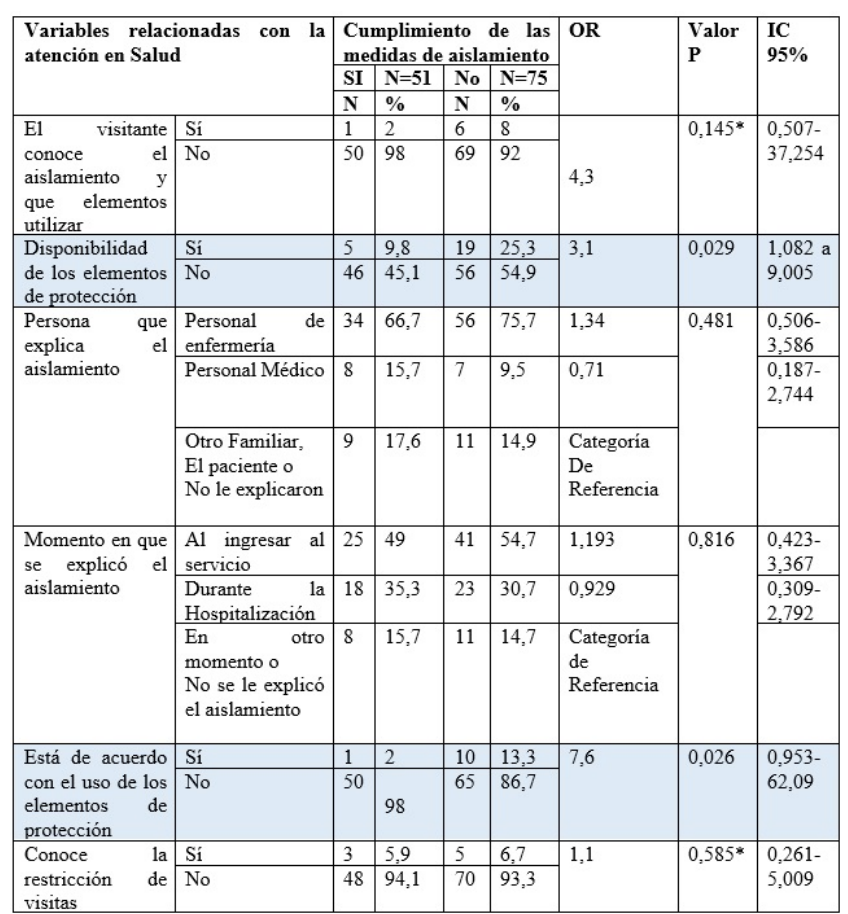

* Se eligió prueba exacta de Fisher por recuento menor que 5 en más de 1 casilla. Fuente: Elaboración propia. 
Mediante regresión logística, se encontró que hay 3,7 veces la probabilidad de que con disponibilidad de los elementos de protección para el aislamiento se cumpla con él y 9,7 veces la probabilidad de que al estar de acuerdo con el uso de los elementos de protección para el aislamiento se cumpla con él. (Ver tabla No. 6)

TABLA NO. 5

Regresión logística, cumplimiento del aislamiento

\begin{tabular}{|l|l|l|l|l|} 
Variables Asociadas & $\mathbf{B i}$ & Valor P & $\begin{array}{l}\text { OR } \\
\text { Ajustado }\end{array}$ & IC 95\% \\
\hline Constante & 0,022 & 0,917 & & \\
\hline Disponibilidad de EPP & 1,313 & 0,016 & 3,717 & 1,28 a 10,81 \\
\hline $\begin{array}{l}\text { Estar de acuerdo con el uso de } \\
\text { los elementos de protección }\end{array}$ & 2,281 & 0,033 & 9,783 & 1,20 a 79,60 \\
\hline
\end{tabular}

Fuente: Elaboración propia.

Según la prueba de Hosmer Lemeshow (Sig. 1,000), el valor P >0,05 indica que las variables se ajustan bien al desenlace. Sin embargo, según el R cuadrado de Nagelkerke $(0,129)$ las dos variables que se muestran en el modelo Anal explican en un $12 \%$ el cumplimiento del aislamiento.

\section{Discusión}

Son varios los factores que llevan a la propagación de microorganismos multirresistentes o de fácil contagio. Los trabajadores de la salud y los visitantes de los pacientes pueden ser agentes silenciosos en la trasmisión de estos y, en especial, cuando incumplen ciertas precauciones como las medidas de aislamiento.

En cuanto a la adherencia en sí de las medidas de aislamiento, muy similar a nuestro estudio, donde el 40,5\% de los visitantes cumplían con el aislamiento, en el de Monsalve C el cumplimiento del aislamiento determinado por el uso correcto de la mascarilla fue del 39,7\% y en el estudio de Seibert G y Col. El 42\% de los participantes cumplieron totalmente con las precauciones de contacto (13), en otras palabras, más de la mitad de los visitantes incumplen con los aislamientos y reaArman la importancia del problema a intervenir.

Se puede ver que existen factores sociodemográAcos y de atención en salud que están relacionados con el cumplimiento de estas precauciones y que algunos estudios demuestran que las personas jóvenes son quienes tienen mayor comprensión de las precauciones de aislamiento (10). Así, se pone en evidencia que es difícil estandarizar esta información a todos los visitantes, lo que motiva a que se den instrucciones mucho más claras a personas adultas y adultas mayores.

Muchas veces el hecho de cumplir con el aislamiento tiene que ver con la persona que lo explica. En el actual estudio, este cumplimiento es un $34 \%$ más frecuente cuando lo explica el personal de enfermería que cuando lo explica otro familiar; también, al mismo paciente o no le fue explicado el aislamiento. Por su parte, es un $19 \%$ más frecuente si la explicación fue al ingreso del servicio que en otro momento. Además, en estudios como el de Monsalve C., más del 50\% de las personas fueron informadas por el personal de enfermería (10). Podríamos decir que la información que brinda el personal de enfermería muchas veces es mejor asimilada y más asequible para los visitantes, por lo que se recomienda a las instituciones brindar más espacios y apoyo en este sentido.

Una diAcultad planteada para el cumplimiento de los aislamientos es la disponibilidad de los elementos de protección oportunamente que, en general, según los estudios, se encuentran bastantes disponibles. En nuestro estudio fue del $81 \%$, en el de Monsalve C. fue del 89,7\% (10), y para el de Seibert G y Col., un 96,8\% de los visitantes estuvieron de acuerdo con que había un acceso adecuado a los EPP (13). Este aspecto tiene una fuerte relación con el hecho de cumplir o no con el aislamiento, lo que indica la importancia de reforzar más su importancia con el personal de salud de los servicios y evaluarlo.

Las precauciones de aislamiento son un tema que se ha aplicado desde tiempo atrás, pero es poco lo que se ha cuestionado sobre el requerimiento de todas estas precauciones y quienes las deben usar. Estudios como el de Seibert plantean que los familiares no cumplían con el aislamiento, ya que opinaban que posiblemente 
estaban colonizados de sus mismos microorganismos al convivir con el paciente (13). En nuestro estudio, el $8,7 \%$ de las personas no estaba de acuerdo con el aislamiento y, por lo tanto, no lo cumplían por razones similares; sería interesante replantear algunas de estas medidas basándose en nuevos estudios.

Por otro lado, la precaución universal del lavado de manos es indispensable, dado que ha demostrado su beneacio en miles de estudios. En nuestro estudio, casi el total de los visitantes (95,2\%) conocía su importancia. En contraste, en un hospital de tercer nivel, en España, mediante la observación directa al personal de salud y visitantes, se encontró una adherencia a la medida del $26,5 \%$ en las monitorizaciones a la entrada a las habitaciones y $35,2 \%$ a la salida (9). En consecuencia, se hacen necesarios más estudios donde se pueda hacer observación directa de los participantes.

Existen otros factores que pueden entorpecer el cumplimiento del aislamiento como la alergia a los guantes de látex y el calor al estar en la habitación del paciente aislado. Estos factores fueron manifestados por los participantes en la investigación de Seibert (13) y en la nuestra, por lo que requieren de mayor exploración y control.

Revisando investigaciones de factores relacionados con el cumplimiento de los aislamientos diferentes a los tratados, se encontró un estudio multicéntrico de regresión logística realizada con datos de hospitales de Francia donde tenían en cuenta la falta de prescripción médica, el tipo de infección y el área donde se encuentra el paciente; igualmente se recalca que el uso de estas precauciones es útil en situaciones de brotes o sin ellos (14).

Dentro de la búsqueda para la mejoría en el cumplimiento de los aislamientos, se debe tener siempre en cuenta la educación al personal de salud, los pacientes y las familias. En Perú, por ejemplo, en un instituto oncológico evaluaron la eAcacia de un programa para la prevención de IAAS y encontraron que el incumplimiento de algunas medidas de bioseguridad fue de hasta el 99,3\%, pero luego de la intervención educativa, la práctica de todas las medidas llegó a más del 70\% (15).

Para investigar sobre el aislamiento por contacto y los factores que favorecen o no su adherencia, es necesario tener en cuenta los recursos de la institución, la disposición y capacitación del personal de salud, las características y concepciones previas de los visitantes, la calidad de información brindada, la persona que explica el aislamiento, el momento en que lo explica, la indicación médica previa, el área donde se encuentra el paciente, entre otros factores que han sido poco explorados. Estos factores motivan a realizar más estudios y a tratar estos temas más frecuentemente en las instituciones de salud con el an de lograr una adecuada adherencia a las medidas de aislamiento.

\section{Conclusiones}

La disponibilidad de elementos de protección para usar en el aislamiento por contacto y estar de acuerdo con el aislamiento del paciente son factores íntimamente relacionados con su cumplimiento. Es necesario, entonces, que las instituciones garanticen suAcientes insumos para los visitantes; de igual manera, el personal de la salud debe mantenerlos a disposición oportunamente y de fácil acceso para ellos; además, es necesario que tanto el personal como los visitantes reciban una mayor sensibilización en este tema.

\section{Referencias}

1. Siegel JD, Rhinehart E, Jackson M, Chiarello L. 2007 Guideline for Isolation Precautions: Preventing Transmission of Infectious Agents in Health Care Settings. Am J infection control. 2007;35(10):S65-164. Disponible en: htt ps://www.cdc.gov/infectioncontrol/guidelines/isolation/index.html

2. Saavedra CH, Ordóñez KM, Díaz JA. Impacto de la infección nosocomial en un hospital de Bogotá (Colombia): efectos en mortalidad y costos. Rev. chil. infectol. 2015;32(1). Feb. http://dx.doi.org/10.4067/S0716-101820 15000200004 
3. OMS. Resistencia a los antimicrobianos [Internet]. [Fecha de última actualización: 15 de febrero de 2018, citado 13 de noviembre de 2019]. Disponible en: https://www.who.int/es/news-room/fact-sheets/detail/resistenciaa-los-antimicrobianos.

4. Álvarez-Lerma F, Gasulla GM, Abad-Peruga V, Pueyo-Pont JM, Tarragó-Eixarch E. Efectividad del aislamiento de contacto en el control de bacterias multirresistentes en un servicio de medicina intensiva. Enfermedades Infecciosas y Microbiología Clínica. 2002;20(2):57-63. https://doi.org/10.1016/S0213-005X(02)72742-0

5. Organización Panamericana de la Salud. Guía de evaluación rápida de programas hospitalarios en prevención y control de las infecciones asociadas a la atención de salud [Internet]. Washington D.C: 2010. Disponible en: ht tps://www.paho.org/hq/dmdocuments/2011/SPA-Guia-Evaluacion-IAAS.pdf

6. Martínez MLO, Duran MEM, García OEP, Bonilla HQ, Instituto Nacional de Salud, Protocolo de vigilancia en Salud Publica: Infecciones Asociadas a Dispositivos 2016. Disponible en: https://cruevalle.org/files/PRO-Infe cciones-asociadas-a-dispositivos.pdf.

7. Cohen CC, Cohen B, Shang J. Effectiveness of contact precautions against multidrug-resistant organism transmission in acute care: a systematic review of the literature. J Hosp Infect. 2015;90(4):275-84. https://doi .org/10.1016/j.jhin.2015.05.003.

8. Munoz-Price LS, Banach DB, Bearman G, Gould JM, Leekha S, Morgan DJ et al. Isolation precautions for visitors. Infection control and hospital epidemiology. 2015;36(7):747-58. https://doi.org/10.1017/ice.2015.67

9. González-Estrada A, Fernández-Prada M, Martínez-Ortegaa C, Lana-Pérez A. y López-González ML. Cumplimiento de las precauciones de aislamiento de contacto por microorganismos multirresistentes en un hospital de tercer Nivel. Rev Calid Asist. 2016;33(8):486-88. http://dx.doi.org/10.1016/j.cali.2016.01.003

10. Monsalve $\mathrm{C}$. Adherencia a técnicas de aislamiento en familiares y visitantes de pacientes aislados por vía aérea. [Tesis de posgrado; digital]. [Medellín]: Universidad de Antioquia. 2016. Disponible en: http://bibliotecadigital.ude a.edu.co/bitstream/10495/5538/1/MonsalveCindy_2016_AdherenciaFamiliaresPacientes.pdf

11.Tamayo M, Echeverry LM. Protocolo de aislamientos hospitalarios. Hospital Universitario San Vicente Fundación, versión 3, julio de 2017.

12. Hospital San Vicente Fundación. Indicadores asistenciales del hospital San Vicente Fundación Medellín creados por el departamento de epidemiología. Aislamientos hospitalarios. Disponible en https://husvp.pensemos.com /suiteve.

13. Seibert G, Ewers T, Barker AK, et al. What do visitors know and how do they feel about contact precautions? Am J Infect Control. 2018;46(1):115-17. https://doi.org/10.1016/j.ajic.2017.05.011

14. Bénet T, Girard R, Gerbier-Colomban S, et al. Determinants of Implementation of Isolation Precautions Against Infections by Multidrug-Resistant Microorganisms: A Hospital-Based, Multicenter, Observational Study. Infect Control Hosp Epidemiol. 2017;38(10):1188-95. https://doi.org/10.1017/ice.2017.153

15. Cuéllar P. de L, Rosales R, Aquiño F. EAcacia de un programa educativo para la prevención y el control de infecciones intrahospitalarias en el Instituto Especializado de Enfermedades Neoplásicas, Lima, Perú. Rev Peru Med Exp Salud Pública. 2004;20(1):37-43. Disponible en: http://www.scielo.org.pe/scielo.php?script=sci_arttext\&pid $=$ S1726-46342004000100007\&lng $=$ es.

\section{Notas}

* Artículo original de investigación.

\section{Licencia Creative Commons CC BY 4.0}

Limitaciones: El estudio no pudo cumplir con una muestra probabilística ya que se realizó durante la pandemia del SARS-COV 2, donde hubo restricción de los visitantes y cierre de servicios; otra limitación fue que solo en algunas ocasiones se pudo realizar una observación directa de la práctica de todas las precauciones 
por contacto de los visitantes, por lo que se requieren más estudios prospectivos donde sea más factible realizarla.

Financiación: El estudio se realizó con recursos de la Universidad CES y del hospital San Vicente Fundación Medellín.

Conflicto de intereses: La autora declara no tener conÁicto de intereses.

Cómo citar este artículo: Arango Castrillón L. Factores asociados con el cumplimiento de las precauciones de contacto en un hospital de alto nivel de complejidad. Investigación en Enfermería, Imagen y Desarrollo. 2021;23. https://doi.org/10.11144/Javeriana.ie23.facp 